\title{
A LATTICE-THEORETIC EQUIVALENT OF THE INVARIANT SUBSPACE PROBLEM
}

\author{
W. E. LONGSTAFF
}

\begin{abstract}
Every bounded linear operator on complex infinite-dimensional separable Hilbert space has a proper invariant subspace if and only if for every lattice automorphism $\phi$ of a certain abstract complete lattice $P$ (defined by N. Zierler) there exists an element $a \in P$ different from 0 and 1 such that $\phi^{2}(a) \leq a$.
\end{abstract}

Let $H$ be a complex, infinite-dimensional, separable Hilbert space. The Invariant Subspace Problem is: does every operator $T \in B(H)$ have a nontrivial invariant subspace? Several equivalent problems are known; some are mentioned in [3] (e.g. p. 190, p. 194), see also [2]. In this note we point out that this famous problem is equivalent to a problem in lattice theory (Corollary 1).

Our equivalence rests on two results. Firstly, the lattice $C(H)$ of all closed subspaces of $H$ has a lattice-theoretic characterization due to Zierler $[\mathbf{4}, \mathbf{5}]$. Secondly, every lattice automorphism $\phi$ of $C(H)$ is spatial in the sense that there exists a bicontinuous linear or conjugate linear bijection (unique up to nonzero scalar multiples) $S: H \rightarrow H$ such that $\phi(M)=S M$ for every $M \in C(H),[\mathbf{1}]$.

Let Aut $\mathrm{C}(H)$ denote the group of automorphisms of $\mathrm{C}(H)$.

THEOREM 1. The following are equivalent.

(1) For every $T \in B(H)$ there exists $M \in C(H)$ different from (0) and $H$ such that $T M \subseteq M$;

(2) For every $\phi \in \operatorname{Aut} C(H)$ there exists $M \in C(H)$ different from (0) and $H$ such that $\phi^{2}(M) \subseteq M$.

Proof. Assume (1) holds. Let $\phi \in \operatorname{Aut} C(H)$ be induced by $S$. Then $\phi^{2}$ is induced by $S^{2}$ which is linear. Thus (2) holds.

Conversely, assume (2) holds. Let $T \in B(H)$ and let $\lambda$ be a scalar satisfying $|\lambda|>\|T\|$. Then $S=T-\lambda$ is invertible and [3, p. 34] $S=R^{2}$ for some invertible operator $R \in B(H)$. If $\phi$ is the automorphism induced by $R$ and $M$ is as in (2) above, we have $S M \subseteq M$ so $T M \subseteq M$.

Let $P$ be an abstract lattice satisfying the (lattice-theoretic) hypotheses of Theorem 2.2 of [4] and assume also that (in the notation of [4]) the coordinatizing division ring $D$ is algebraically closed. Then there exists a lattice isomorphism $\theta: P \rightarrow C(H)$ (satisfying $\theta\left(a^{\prime}\right)=\theta(a)^{\perp}$, where $a^{\prime}$ denotes the complement of $a$ in $P$ ). Let Aut $P$ denote the set of automorphisms of $P$, and let 0 (respectively, 1) denote the least (respectively, greatest) element of $P$.

Received by the editors July 8,1985 .

1980 Mathematics Subject Classification (1985 Revision). Primary 47A15; Secondary 06B05. 
COROLLARY 1. The following are equivalent.

(1) For every $T \in B(H)$ there exists $M \in C(H)$ different from (0) and $H$ such that $T M \subseteq M$;

(2) For every $\phi \in$ Aut $P$ there exists $a \in P$ different from 0 and 1 such that $\phi^{2}(a) \leq a$.

Let $\mathcal{L}$ denote the set of automorphisms of $\mathcal{C}(H)$ that are induced by invertible operators of $B(H)$. Obviously $\mathcal{L}$ is a subgroup of Aut $C(H)$. Some characterizations of $\mathcal{L}$ follow.

THEOREM 2. $\mathcal{L}=\left\{\phi^{2} \psi^{2}: \phi, \psi \in \operatorname{Aut} C(H)\right\}$.

Proof. Let $\mathcal{Q}=\left\{\phi^{2} \psi^{2}: \phi, \psi \in \operatorname{Aut} \mathcal{C}(H)\right\}$. Clearly $\mathcal{Q} \subseteq \mathcal{L}$. Let $\eta \in \mathcal{L}$ and suppose that $\eta$ is induced by $S \in B(H)$. Then $S=U A$ with $U$ unitary and $A$ positive and invertible. There exists $V \in B(H)$ such that $V^{2}=U$. Also $\left(A^{1 / 2}\right)^{2}=A$. Thus $\eta=\phi^{2} \psi^{2}$ where $\phi$ is induced by $V$ and $\psi$ is induced by $A^{1 / 2}$.

COROLlaRY 2. $\mathcal{L}$ is the subgroup of Aut $\mathcal{C}(H)$ generated by $\left\{\phi^{2}: \phi \in \operatorname{Aut} C(H)\right\}$.

If $G$ is an abstract group, it is clear that a subgroup $K$ of $G$ has index 2 if and only if $g h \in K$ whenever $g, h \notin K$. Also, every subgroup of $G$ of index 2 is a maximal proper (normal) subgroup and contains the square of every element of $G$. From these observations and the preceding corollary it follows that $\mathcal{L}$ is the only subgroup of Aut $C(H)$ of index 2 .

\section{REFERENCES}

1. P. A. Fillmore and W. E. Longstaff, On isomorphisms of lattices of closed subspaces, Canad. J. Math. 36 (1984), 820-829.

2. E. A. Nordgren, H. Radjavi, and Peter Rosenthal, $A$ geometric equivalent of the invariant subspace problem, Proc. Amer. Math. Soc. 61 (1976), 66-68.

3. H. Radjavi and Peter Rosenthal, Invariant subspaces, Springer-Verlag, New York, 1973.

4. N. Zierler, Axioms for nonrelativistic quantum mechanics, Pacific J. Math. 11 (1961), 1151-1169.

5. __ On the lattice of closed subspaces of Hilbert space, Pacific J. Math. 19 (1966), 583-586.

Department of Mathematics, University of Western Australia, Nedlands 6009, AUSTRALia 